Aus dem städtischen Siechenhause zu Frankfurt a. M. (Direktor: Prof. Dr. August Knoblauch.)

\title{
Ein Fall von multipler Sklerose, kompliziert durch eine chronische Geistesstörung.
}

Von

AUGUST KNOBLAUCH.

Auf der 30. Versammlung südwestdeutscher Irrenärzte (Frankfurt a. M., 19. November 1899) habe ich unter der Diagnose , M M $u$ tiple Neuritis und abgelaufene Korsakowsche Psychose" eine Kranke des städtischen Siechenhauses vorgestellt $\left.{ }^{1}\right)$. Ihr Leiden hat während seiner 25 jährigen Dauer zu verschiedenen Zeiten ganz verschiedene Zustandsbilder geboten, und dies hat die einzelnen Ärzte, die die Kranke in dieser langen Zeit behandelt oder begutachtet haben, zu ganz verschiedenen Auffassungen der Krankheit geführt. Der weitere Verlauf des Leidens hat auch meine anfängliche Diagnose als unrichtig erwiesen und hat mich veranlasst, eine multiple Sklerose anzunehmen; nachdem nun diese Diagnose durch die Obduktion der am 9. Dezember 1907 verstorbenen Kranken bestätigt worden ist, ist eine Richtigstellung meiner früher ausgesprochenen Ansicht durch Mitteilung der Krankengeschichte und des Obduktionsbefundes dieses eigenartigen Falles geboten.

die über den Kontraktionsgrad berichtenden, von ihm sogenannten ,.Innervationsmerkmale" dem Kleinhirn und von da clem Grosshirn zuströmen lässt. Indem er aber mit Gründen, die mir nicht zureichend erscheinen, jene peripheren ,Innervationsmerkmale“ für ihrem Wesen nach unbewusste Eindrücke erklärt, entzieht sich für ihn deren Störung,,vollständig der klinischen Prüfung", und jene Theorie der Kleinhirnfunktion wird bloss per exclusionem aufgestellt. Sobald man dagegen in der fein abgestuften Schwere-Empfindung einen bewussten Anteil jener "Innervationsmerkmale" anerkennt, werden diese sehr wohl zum Gegenstand klinischer Untersuchung, und es wird damit auch jene Theorie erst einer direlsten empirischen Begründung zugänglich.

$\left.{ }^{2}\right)$ Neurologisches Zentralblatt. 18. Jahrgang. 1899. S. 1141. 
Frau Louise E., Kaufmannswitwe, geboren am 8. XII. 1843, wurde am 11. II. 1895 in das hiesige Armenasyl aufgenommen, aus dem im Jahre 1898 das städtische Siechenhaus hervorgegangen ist. Als ich damals die ärztliche Leitung der neuerrichteten Anstalt übernahm, waren eine Krankengeschichte oder irgend welche anamnestischen Anhaltspunkte nicht vorhanden. Die Kranke wurde in den Journalen des Asyls seit ihrer Aufnahme unter der Diagnose ,rechtsseitige Hemiplegie und postapoplektische Demenz" gefülurt. Bei dem eigenartigen und unklaren Zustandsbild, das Frau E. indessen damals nach bereits 16 jährigem Verlaufe ihres Leidens bot und durch das die angeführte Diagnose nicht gerechtfertigt wurde, schien es mir notwendig, den langjährigen Verlauf der Krrankheit tunlichst zu rekonstruieren und die eigenen Angaben der Kranken durch aktcnmässige Belege zu kontrollieren. Meinen damaligen Erhebungen haben zugrunde gelegen:

1. Die ärztlichen Akten einer auswärtigen öffentlichen Irrenanstalt, die mir von deren Direktion in liebenswürdigster Weise zur Verfügung gestellt worden sind. Sie enthalten u. a. den für unsere Kenntnis über die Entwickelung des Leidens wichtigen Fragebogen zur Aufnahme der Kranken vom 30. I. 1883 ,

2. die Journale des Königlichen Kreisphysikats,

3. die Entmündigungsakten,

4. die Akten des hiesigen Magistrats-Armenamtes.

Freilich ist diese auf Grund des angeführten Aktenmaterials nachträglich erhobene Anamnese und rekonstruierte Krankengeschichto angesichts der langen Dauer des Leidens naturgemäss lückenhaft geblieben; immerhin ist es aber gelungen, hinsichtlich einzelner Perioden der Krankheit sichere Anhaltspunkte für die damals vorhandenen Symptome oder wenigstens zusammenfassende Urteile über das jeweilige Zustandsbild zu erhaiten.

Eine erbliche Belastung der Kranken liess sich nicht feststellen. Thr Vater, ein Kaufmann, ist 1848 der politischen Verhältnisse wegen nach Nordamerika ausgewandert und dort im Sezessionskriege 1863 gefallen. Ihre Mutter ist frühzeitig gestorben, als die Kranke etwa 2-3 Jahre alt war (Todesursache unbekannt). Frau E. wurde nach dem Tode ihrer Mutter von deren Schwester erzogen und kam nach ihrer Konfirmation in ein klösterliches Institut nach Paris, von wo sie nervös und chlorotisch in die Heimat zurückkehrte. Sie galt für gelehrig, schnellfassend, von gutem Gedächtnis und sehr talentvoll.

Zweimal ist die Kiranke verheiratet gewesen (23. XII. 1865-18. IV. 1866 und 28. IV. $1870-4$. IV. 1891). Die erste Ehe, von nur viermonatlicher Dauer, ist kinderlos geblieben; in der zweiten Ehe sind auf einen Abort die Geburten von zwei Kindern (1873 und 1878) gefolgt. Beide (Töchter) sind gesund. Die Kranke ist vom 13. bis 48 . Lebensjahre regelmäissig menstruiert gewesen; nur kurz nach dem Ausbruch der Psychose waren die Menses vorübergehend profuser und häufiger als sonst. Ernste Krankheiten sind, von einer hartnäckigen Gesichtsneuralgie während der zweiten Schwangerschaft abgesehen, nicht beobachtet-worden. Wohl aber ist Frau E. im Beginn der Erkrankung hochgradig blutarm gewesen. Lues und Potus sind ausgeschlossen.

Nachdem seit. Anfang 1882 heftige Schmerzen im rechten Arm aufgetreten waren (vom Arzt als schwere ,Neuralgia brachialis" bezeichnet), entwickelte sich im Juli desselben Jahres anscheinend olne erkennbare Ursache und ziemlich rasch eine psychische Störung. Unter Gehörshalluzinationen schreckhaften Inhalts trat ein Zustand ängstlichen Affektes auf. Die Kranke glaubte sich verfolgt und bedroht: sie fïrchtete, umgebracht zu werden; hörte klopfen und die Vorbereitungen zu ihrer Ermordung treffen. Sie wurde zeitweilig sehr erregt und suchte $\%$ entfliehen oder sich in ihr Zimmer einzuschliessen, um vor ihren Verfolgern sicher zu sein. Bald zeigte sich auch eine auffällige Störung der Ideenverknüpfung und des Gedächtnisses. Dieser Zustand der Erregung hielt indessen nicht lange an; nach einigen Wochen ging er in einen ausgesprochenen depressiven Affekt mit isolierten deliriösen Ideen, Illusionen und Halluzinationen über. Meist war 
die Franke ganz apathisch, ohne sich zu beschäftigen und ohne anf Amreden zu reagieren; nur zeitweise traten vorübergehende, heftige Errogungszustiinde auf. Gleichzeitig wurcle ein Tremor der Hände und Inliontinenz beobachtet. Bald traten Nalrungsverweigerung und Taedium vitae hinzu. Wiedorholt versuchte die Kranke, sich aus dem Fenster zu stürzen; sie drehte Tücher strickartig zusammen, um sich zu erdrosseln, und nur durch eine ständige Ueberwachung war sie davon abzuhalten, sich die Pulsadern am Vorderar'm zu öffnen, wozu sie selbst ilure Zähne zu benützen suchte.

Dieser intensive Suizidaldrang veranlasste am 22. März 1883 die Ueberfiihrung der Kranken in die Irrenanstalt in einem Zustand ,tiefer, stuporöser Melancholie." Wie aus einem Briefe des Direktors an die Angehörigen rom 25. Mai 1883 ersichtlich ist, trug , das bei der Aufnahme 'bereits voll entwickelte Krankheitsbild in all seinen Schatticnungen das Gepräge geistiger Schwäche." Am auffälligsten war die schwere Störung des Gedächtnisses, infolge deren die Krranke die Ärzte trotz ihrer täglichen Besuche nicht kannte und nicht wieder erkannte, und des Urteils, sowie die rasche Verblödung der Iirmuken.

Die Nachrichten über den weiteren Verlauf des Leidens während des Aufenthaltes der Kranken in der Anstalt sind leider spizilich. Sie ist am 22. X. 1883 ungeheilt und mit den fritheren Suizidideen auf Ver]angen ihres Gatten provisorisch entlassen und in Privatpflege nach Frankfurt a. M. überführt worden. Hier ist sie bis zu ihrer Aufnalme in das Armenasyl geblioben. Das drei Jahre spä.ter (XI. 1886) ausgestellte Attest eines hiesigen Arztes berichtet, dass Frau E. mnhoilbar geisteskrank und unfähig zu irgend einer verantwortlichen Handlung sei.

NäIleres über die Art der Geistesstörung erfahren wir erst aus einem ausfülrlichen Gutachten, das am 15. V. $1890 \mathrm{zur}$ Einsetzung der Pflegschaft erstattet worden ist. Damals bot Frau E. nach der Schilderung des Gutachters das Bild einer manischen Erregung: gehobene Stinmung mit erotischer Fäbung; Rededrang, begleitet von lebhaftem Mienen- und Geberdenspiel; Projektemacherei; gesteigerter Bewegungsdrang; triebartiges Sammeln aller möglichen bunten und glänzenden Gegenstönde, die zu Schmucksachen der verschiedensten Art, zu Olnringen, Halsbändern u. dergl., verarbeitet wurden; Bekritzeln der Wände mit allerlei kindlichen Zeichnungen, oft sexuellen Charakters; läppisches, oft unzüchtiges Wesen; Schlaflosigkeit usw. Dabei wird das Gedächtnis ausdrücklich als, ,recht gut" bezeichnet. Bezüglich somatischer Erscheinungen wird festgestellt, dass clie Kranke über Nervenschmerzen in den Armen und Beinen klagte, deren Ursache unklar blieb. Dieser manische Zustand scheint längere Zeit angehalten zu haben; wenigstens besagt ein zur Einleitung der Entmündigung erstattetes Gutachten desselben Sachverständigen vom 29. VI1. 1891, dass eine Aenderung in dem Befinden der Kranken nieht eingetreten sei: ,Frau E. ist von nervösen Schmerzen in den Beinen abgesehen, füi deren Fintstehums die Untersuchung keinen Anhaltspunkt gibt, körperlich gesund, dagegen blödsinnig und unfähig, selbst zu hancleln." "Auf Grund chieses Gutachtens ist die Kranke am 30. VII. 1891 entmündigt worden.

Im weiteren $V$ erlaufe des Leidens ist die Stimmung wieder umgeschlagen. In den Aliten befindliche Aussagen von Laien enthalten die Angabe, dass zu Fnde des Jahres 1894 eine ausgesprochene Depression mit Taedium vitae und mit Suizidideen bestanden hat, die indessen, wenigstens in der anfänglichen Intensität, von nicht langer Dauer - mehrere Monate gewesen zu sein scheint, und dass die geistige Schwäche allmählich mehr und mehr zunahm.

Nachdem die Pflegefrau, die Frau E. bei sich aufgenommen und zwölf Jahre lang verpflegt hatte, 1895 gestorben und ihr Haushalt aufgelöst worden und vorher (1891) auch der Gatte der Kranken verstorben war, wurde sie am 3. IT. 1895 wegen ,Ir'rsinnigkeit" und wegen Mangels an genügender Pflege und Aufsicht dem Polizeigewahrsam übergeben und von dort am 11. II. 1895 in das Armenasyl überfüint, nachdem der Polizeiarzt die Internierung der Iranken in einer In'enanstalt nicht für notwendig erachtet hatte. Aus seinem kurzen Bericht vom 6. I1. 1895 ist zu entmehmen, 
dass Frau E. damals volle Einsicht in ihre Lage hatte. Sie machte ïber die Verhältnisse der verstorbenen Pflegefrau richtige Angaben, und eine ausgesprochene geistige Störung konnte nicht mehr nachgewiesen werden. Jedoch wird die Kiranke in dem Bericht als ,,geistesschwach" bezeichnet.

Kurze Zeit später, am 26. III. 1895, berichtet der Arzt des Asyls über eine lähmungsartige Schwäche des rechten Arms und Beins, die er - in völliger Unkenntnis des früheren Verlaufs der Ḱrankheit - als die Folge eines vor langen Jahren erlittenen Schlaganfalls aufgefasst hat. Die Kranke ,ist insofern geistesgestört, als sie mit einem schweren Rückenmarksleiden behaftet zu sein glaubt". Der somatische Befund zusammen mit der vorhandenen geistigen Schwäche und der angeführten Aeusserung, die als hypochondrische Vorstellung gedeutet wurde, veranlasste den Arzt, eine sekundäre „Demenz mit Hemiplegie nach Apoplexie“ anzunehmen.

Aus der nächstfolgenden Zeit liegt das ausführliche Gutachten eines psychiatrischen Sachverständigen vom 27. VI. 1895 vor, das nicht nur in psychischer, sondern auch in somatischer Beziehung die beobachteten Krankheitserscheinungen sorgfältig berücksichtigt. Festgestellt wurde: schwächere Innervation der rechten Cesichtshälfte; leichte Sprachstörung (etwas verlangsamte, schleppende Sprache) mit gelegentlichem Zucken der Mundmuskeln; Schwäche der rechten Hand mit Atrophie der Kleinfingerballenmuskulatur; Zittern der Finger bei ausgestreckten Händen; langsamer schleppender Gang mit Frhöhung der Kniephänomene und Muskelsteifigkeit; sowie auf psychischem Gebiet: stumpfes, teilnahmsloses Wesen: Schwäche des Gedächtnisses für die Vorgänge der letzten Vergangenheit: mangelhaftes Crteil; Euphorie; hypochondrische Sensationen und Vorstellungen. Der Gutachter kommt zu dem Schluss: „Das Krankheitsbild setzt sich mithin zusammen aus Erscheinungen körperlicher Lähmungen, die vom Zentralnervensystem ausgehen, und einer eigentümlichen, die gesamte geistige Tätigkeit umfassenden, geistigen Schwäche, welche namentlich durch die damit verbundene Euphorie als die für die Dementia paralytica charakteristische gekennzeichnet wird."

Aus einem zweiten Gutachten desselben Sachverständigen, 21/2, Jahre später, am 11. XI. 1897 erstattet, ist ersichtlich, dass die Erkrankung nicht den weiteren Verlauf genommen hat, der von einer progressiven Paralyse zu erwarten gewesen wäre. Vor allem hatte in dieser ganzen Zeit die geistige Schwäche der Kranken nicht merkbar zugenommen. Von einer auffälligen Euphorie und von hypochondrischen Vorstellungen wird nicht mehr berichtet. Wohl aber waren körperliche Lähmungserscheinungen an den rechtsseitigen Extremitäten und die Muskelatrophie an der rechten Hand deutlich stärker geworden und auch am rechten Bein ein geringer Muskelschwund hinzugetreten. Die frühere Diagnose wird in dem zweiten Gutachten nicht mehr aufrecht erhalten, an ihre Stelle aber auch keine andere Diagnose gesetzt.

Ein halbes Jahr später, im Juni 1898 , ist die Kranke zum ersten Male von mir untersucht worden. Dabei liess sich nur eine geringe geistige Schwäche feststellen. Die Angaben der Kranken über ihr Vorleben, über den Beginn ihres Leidens und ihre späteren Schicksale haben sich bei der Kontrolle durch die aktenmässigen Belege als vollständig richtig erwiesen. Dagegen war es sehr auffällig, dass das Gedächtnis der Kranken für die einzelnen Perioden ihres Lebens ein verschieden gutes war. Fïr ihre Kindheit und Jugend, sowie für die ganze Zeit vor dem Ausbruch der Y'sychose erwies sich ihr Gedächtnis als ausgezeichnet; für die nächsten Monate war ihre Erinnerung mehr oder weniger getriibt; sie war nahezu vollständig erloschen fïr die ganze Zeit ihrer Verpflegung in der Irrenanstalt und recht liickenhaft fiir die nächsten sieben Jahre. Erst etwa vom Jahre 1890 an zeigte sich das Gedächtnis der Kranken wieder mehr oder weniger vollständig erhalten unä auch die noch 1895 beobachtete Vergesslichkeit in bezug auf die Vorgänge der letzten Vergangenheit liess sich nicht mehr feststellen. Vielmehr war die Merkfähigkeit der Kranken recht gut; ja, sie schien sich in cier Folgezeit noch eher zu bessern, vielleicht durch die Schulung, zu der der regelmässige Verkehr mit den Aerzten der früher sich selbst überlassenen Kranken Gelegenheit gegeben hat. 
Auch hypochondrische Vorstellungen wurden nicht mehr beobachtet. Wenigstens sind die Befürchtungen, die die Kranke hinsichtlich ihres $\mathrm{Zu}$ standes äusserte, von mir nicht als ,hypochondrische" aufgefasst worden, weil ich ihre unklare Vorstellung, an einer schweren und fortschreitenden Erkrankung zu leiden, für berechtigt halten musste.

Die Stimmungslage, die die Kranke zu der Zeit bot, in der ich sie kennen lernte, wurde damals von mir für normal gehalten. Später überzeugte ich mich indessen, dass sie eine leicht deprimierte gewesen ist. Denn zu Ende des Jahres 1898 trat allmählich ein Umschlag der Stimmung ein; die stille, teilnahmlose Kranke wurde lebhaft und heiter, in einer Weise, die als völlig normal imponieren musste. Im Herbst 1899 hat jedoch wieder ein krankhafter Umschlag der Stimmung eingesetzt und diesmal in unverkennbarer Weise nach der manischen Seite hin. Die Kranke wurde auffallend geschwätzig, zeigte eine gewisse Ueberteibung des Wertes der eigenen Persönlichkeit, eine Putzsucht u. dergl. Diese leichte manische Erregung klang nach mehrmonatlicher Dauer ganz allmählich ab. Ein weiterer Wechsel in der Stimmungslage der Kranken wurde nicht mehr beobachtet; zurück blieb neben der geringen geistigen Schwäche eine leichte Euphorie, die trotz allmählicher Zunahme der körperlichen Beschwerden bis zum Tode der Kranken angehalten hat.

Auf somatischem Gebiet wurde im Juni 1898 folgender Nervenstatus aufgenommen:

Pupillen kreisrund, gleichweit, von mittlerer Weite, verengen sich prompt bei Lichteinfall (direkt und konsensuell) und bei Konvergenzbewegungen der Bulbi. Andeutung von Nystagmus. Innervation der äusseren Augenmuskeln intakt. Sehschärfe $6 / 6$. Augenhintergrund und Gesichtsfelder normal. Leichte Parese des rechten Mundfazialis. Keine Störung der Gehirnṇerven; keine Artikulations- oder sonstige Sprachstörung.

Motilität des rechten Arms etwas behindert, insofern die Kranke ausserstande ist, den Arm im Schultergelenk ganz hoch zu heben. Kraft des rechten Arms und der Hand wesentlich vermindert. Muskulatur des Arms im ganzen atrophisch (Masse: $15 \mathrm{~cm}$ oberhalb des Olekranons $\mathrm{r} .=23 \mathrm{~cm}$, l. $=261 / 2 \mathrm{~cm} ; 22 \mathrm{~cm}$ oberhalb desselben $\mathrm{r} .=251 / 2 \mathrm{~cm}, \mathrm{l}=28 \mathrm{~cm}$; oberes Drittel des Unterarms r. $=193 / 4 \mathrm{~cm}, 1 .=21 \mathrm{~cm} ;$ Mitte desselben $\mathrm{r} .=161 / 2 \mathrm{~cm}$, 1. = $171 / 4 \mathrm{~cm}$; unteres Drittel desselben r. $=14 \mathrm{~cm}, 1 .=143 / 4 \mathrm{~cm}) . \quad B e-$ sonders auffällige Atrophie an den Muskeln des Daumen und Kleinfingerballens wie auch der $\mathrm{Mm}$. interossei und lumbricales der rechten Hand. Deutliche Herabsetzung der galvanischen und faradischen Erregbarkeit der atrophischen Muskeln von den Nerven aus; bei direkter faradischer Reizung etwas träge, wurmförmige Muskelzuckungen. Keine Ea R. Sehnenund Periostreflexe am rechten Arm sehr lebhaft. Taktile Sensibilität und Temperaturempfindung anscheinend intakt bei leichter Hyperalgesie. Spontan und bei Druck auf die Nervenstämme rechts Schmerzen in Arm und Hand; Paraesthesien (Ameisenlaufen) in der rechten Hand.

Motilität und grobe Kraft des linken Arms und der Hand normal. Keine Atrophie der Muskulatur. Sehnen- und Periostreflexe in normaler Stärke vorhanden, etwas weniger lebhaft als rechts. Taktile Sensibilität, Temperatur- und Schmerzempfindung ohme Störung. Nervenstämme nicht druckempfindlich.

An beiden Händen geringer Tremor der Finger, der indessen bei intendierten Bewegungen nicht zunimmt. Keine Störung der Koordination an den oberen Extremitäten.

Ausgesprochene Parese des rechten Beins, das beim Gehen steif gehalten, nachgeschleift und zirkumduziert wird. Gang langsam, schleppend. Rechts geringe Spitzfusstellung. Muskeltonus rechts anscheinend etwas stärker als links; keine ausgesprochenen Spasmen. Geringe Atrophie der Glutaealmuskulatur ohne Ea R. Motilität und grobe Kraft des linken Beins normal. Beiderseits Kniephänomene in normaler Stärke vorhanden; kein Unterschied zwischen rechts und links. Kein Partellarklonus. Achillessehnenreflexe beiderseits nicht auszulösen; kein Fussklonus. Sohlenreflexe beiderseits nicht 
auszulösen. Alle Qualitäten der Sensibilität an den Unterextremitäten intakt. Zeitweise schmerzhaftes Ziehen in beiden Waden; taubes Gefühl in beiden Beinen. Mässige Druckschmerzhaftigkeit der Nervenstämme beiderseits. weisbar.

Keine Störung der Koordination; Rombergsches Symptom nicht nach-

Bauchhautreflexe beiderseits nicht auszulösen.

Funktion von Blase und Mastdarm normal.

Auffällig war ferner ein oft beobachtetes einseitiges Erröten der paretischen rechten Gesichtshälfte und das üppige, kaum ergraute Kopfhaar der 55 jährigen Kranken. Nach ihrer glaubwürdigen Angabe hat sie seit 1894 beobachtet, dass ihr damals schon lichtes Haar plötzlich dichter zu werden und zu wachsen anfing. Die Länge des Haares von der vorderen Haargrenze an der Stirn gemessen betrug $1,19 \mathrm{~m}$.

Nachdem der Zustand der Kranken in den ersten Monaten der Beobachtung im Siechenhause stationär geblieben war, trat im Sommer 1899 ziemlich plötzlich, schubartig, eine Zunahme der Beschwerden(Verminderung der Kraft im rechten Arm, Verschlechterung des Ganges, stärkere Schmerzen, auch in den linksseitigen Extremitäten) und eine objektiv nachweisbare Verschlimmerung des Leidens ein.

Der rechte Arm konnte nur noch mit Mühe bis zur Wagerechten erhoben werden; in der Ruhe hing er schlaff, nur im Ellbogengelenk mässig flektiert, herab. Die Beweglichkeit der rechten Hand und der Finger wurde durch die zunehmende Parese und Muskelatrophie immer mehr beschränkt. Die Sehnen- und Periostreflexe verloren allmählich ihre frühere Lebhaftigkeit. Aber auch am linken Arm und namentlich an der Hand liess sich bei erhaltener Motilität eine Verminderung der groben Kraft und eine beginnende, deutliche Atrophie der Muskulatur zunächst am Daumen-, später auch am Kleinfingerballen nachweisen, ohne dass eine Aenderung der elektrischen Erregbarkeit der atrophierenden Muskulatur festzustellen gewesen wäre. Gelegentlich wurden fibrilläre Zuckungen an den kleinen Handmuskeln beiderseits beobachtet. Auch die Nervenstämme des linken Arms erwiesen sich häufig druckempfindlich, wenn auch in geringerem Masse als rechts.

Der Gang der Krranken wurde schwerfälliger. Das rechte Bein wurde noch stärker nachgeschleift und zirkumduziert als früher; aber auch das linke Bein wurde etwas steif aufgesetzt, und auch subjektiv empfand die Kranke sehr wohl, dass es schwächer und schwerer geworden war. Die Schmerzen im linken Bein traten häufiger und heftiger auf und die Nervenstämme waren äusserst druckempfindlich geworden. Bei wiederholter Prüfung der Sensibilität liess sich an allen vier Extremitäten eine Hypcraesthesie der Haut für taktile Reize nachweisen, die auf der rechten Seite etwas stärker zu sein schien als links.

Die im Juni 1898 beobachtete Andeutung von Nystagmus war nur eine vorübergehende Erscheinung gewesen und nicht mehr nachzuweisen.

Es fand sich also in der Hauptsache auf somatischem Gebiet eine Parese aller vier Extremitäten mit Muskelschwund an den Händen und an der rechtsseitigen Glutealmuskulatur, Hyperästhesie der Haut und Druckschmerzhaftigkeit der Nervenstämme. Alle Krankheitserscheinungen waren auf der rechten Körperseite wesentlich stärker und am stärksten wiederum rechterseits am Arm und namentlich an der Hand ausgebildet.

Diese Befunde veranlassten mich zurzeit, als ich die Kranke auf der Versammlung südwestdeutscher Irrenärzte vorgestellt habe (1899), das Vorhandensein einer peripheren multiplen Neuritis anzunehmen. Mit dieser Annahme schienen mir der Beginn und der ganze Verlauf des langjährigen Leidens, soweit die subjektiven 
Beschwerden der Kranken verwertet werden konnten, in vollem Einklang zu stehen. Dem Ausbruch der psychischen Störung waren heftige Schmerzen im rechten Arm vorausgegangen, die von dem behandelnden Arzte als "Neuralgia brachialis" bezeichnet worden waren. Derartige Nervenschmerzen haben die Kranke wie sie stets mit aller Bestimmtheit angegeben hat - seit jener Zeit niemals mehr ganz verlassen; sie haben allmählich, in wechselnder Intensität, auch die übrigen Extremitäten befallen. Die Gutachten aus den Jahren 1890 und 1891 rubrizieren diese ,Schmerzen“" bereits als in den Armen und Beinen bestehend. In den Berichten des Jahres 1895 wird sodann eine Parese im rechten Arm mit Atrophie der kleinen Handmuskeln und eine lähmungsartige Schwäche im rechten Bein erwähnt. Im Herbst 1897 ist eine geringe Atrophie der Muskulatur des rechten Beins hinzugetreten, im Sommer 1899 ein deutlicher Muskelschwund an der linken Hand und eine Parese des linken Beins. Zudem bestand eine Druckschmerzhaftigkeit der Nervenstämme an allen vier Extremitäten.

Von der Annahme einer multiplen Neuritis ausgehend, war ich nun auch zu einer ganz anderen Auffassung der bei der Kranken 18 Jahre zuvor aufgetretenen Psychose gelangt als die früheren Gutachter. Im Jahre 1895 war die Krankheit ausdrücklich als ,postapoplektische Demenz" und als ,progressive Paralyse" bezeichnet worden. In den Berichten aus den früheren Jahren ist eine bestimmte Diagnose nicht ausgesprochen; doch ist aus dem Wortlaut der Gutachten zu schliessen, dass die Geisteskrankheit der Frau E. im Jahre 1882 als Verfolgungswahn, 1883 als progressive Paralyse (?), 1890 als Manie und 1894 als Melancholie aufgefasst worden ist. Ich selbst habe im Jahre 1899 unter Berücksichtigung des somatischen Befundes und des ausgesprochenen Verlustes der Erinnerung an die ersten sieben Jahre der Erkrankung eine abgelaufene Korsakowsche Psychose angenommen. Ein ätiologisches Moment habe ich freilich nicht anzugeben gewusst, indem Alkoholmissbrauch mit Sicherheit ausgeschlossen werden konnte und auch andere akute oder chronische Intoxikationen nicht nachzuweisen waren.

Im Laufe der letzten acht Jahre sind bei Frau E. weitere psychische Störungen nicht mehr beobachtet worden. Vielmehr war nur eine geringe mit Euphorie verbundene geistige Schwäche vorhanden, die wohl mit der Zeit noch immer etwas stärker geworden ist, keineswegs aber zu einer so hochgradigen Verblödung geführt hat, wie man sie bei der progressiven Paralyse nach mehrjähriger Dauer des Leidens zu sehen gewohnt ist.

Auf körperlichem Gebiet hat die Krankheit dagegen langsam, aber stetige Fortschritte gemacht, die wiederholt unter deutlich erkennbaren Schiiben erfolgt sind. Dabei erwiesen sich manche Krankheitserscheinungen anfangs nur von passagerer Dauer, bis sie nach wiederholtem Verschwinden und Wiederauftreten endlich ständig nachzuweisen waren, während andere Symptome nur vorübergehend zur Beobachtung gekommen sind.

Eine fortschreitende Verschlimmerung zeigten die Lähmungserscheinungen an den Armen und Beinen und die Atrophie der Muskulatur. Besonders am rechten Bein nahm die grobe Kraft mehr und mehr ab, so dass 
die Kranke genötigt war, beim Stehen und Gehen die Last des Körpers vorzugsweise auf dem linken Bein zu tragen. Die Atrophie der Gefäss- und Rollmuskulatur des rechten Obersehenkels (besonders der Mm. glutaeus medius und gl. maximus) war schliesslich so stark, dass man mit der Hand tief in die Fossa ischiadica eindrücken und von hier aus die Innenfläche des Femur abtasten konnte. Infolge dieses hochgradigen Muskelschwundes war die Fixierung des rechten Beins im Hüftgelenk so mangelhaft, dass beim Gehen leicht unbeabsichtigte Rotationsbewegungen des Oberschenkels nach innen oder aussen zustande kamen. Hierdurch war die Aufrechterhaltung des Gleichgewichtes in holiem Masse erschwert, so dass die Kranke beim Gehen durch Bewegungen der Arme die Balanze unterstiitzen musste. Viel geringer blieben Parese und Muskelschwund am linken Bein. Hier war eine besonders starke Atrophie des M. vastus medius vorhanden, so dass im Gebiet desselben die innere Kontur des Oberschenkels eine leichtkonkav verlaufende Linie aufwies.

Während die Muskelatrophie an Arm und Hand der rechten Seite nahezu unverändert blieb und an der linken Hand nur langsame und geringe Fortschritte machte, trat im Spätsommer 1900 ziemlich schnell eine Atrophie der Muskulatur des linken Oberarms ein. Sie entwickelte sich indessen bis zu dem Tode der Kranken nicht zu soleher Intensität, wie sie die Muskelatrophie am rechten Oberarm bereits 1898 erreicht hatte. (Masse am 18. XI. 1900: $15 \mathrm{~cm}$ oberhalb des Olekranons $\mathrm{r} .=23 \mathrm{~cm}, 1 .=24 \mathrm{~cm} ; 22 \mathrm{~cm}$ oberhalb desselben $\mathrm{r} .=25 \mathrm{~cm}, 1 .=261.2 \mathrm{~cm}(\mathrm{ef} . \mathrm{S} .242)$ ). In der Folgezeit stellte sich auch eine Atrophie der Mm. deltoideus und pectoralis major der rechten Seite ein, wodurch die Motilität im Schultergelenk noch mehr eingeschränkt wurde. In sämtlichen atrophischen Muskeln liess sich schliesslich Ea $R$, nachweisen.

In den nicht atrophischen Muskeln trat allmählich eine Hypertonie ein, anfangs in bezug auf die Stärke wechselnd, bis sie später zu ausgesprochenen Spasmen fülnrte. Indessen erreichten die Spasmen erst im Laufe des letzten Jahres der Erkrankung eine stärkere Intensität von bleibender Dauer und zwar nur an der Muskulatur des rechten Beins, während sie an Arm und Bein der linken Seite nur vorübergehend stärker waren und am rechten Arm dauernd fehlten.

Dementsprechend war auch dasVerhalten der Selnnenreflexe wechselnd. Während der Tricepsreflex und die Periostreflexe an den Unterarmen anfangs rechts viel lebhafter waren als links, liess sich später eine deutliche Differenz zwischen beiden Seiten nicht mehr feststellen; es blieben die Reflexe an den oberen Extremitäten etwa in normaler Stärke vorhanden. Die Kniephänomene waren jahrelang beiderseits gleichstark, etwas lebhaft, aber innerhalb der normalen Breite. Erst 1906 trat eine allmählich deutlicher werdende Differenz in der Stärke der Reflexe in die Erscheinung, indem links eine Steigerung des Kniephänomens nachweisbar wurde. Zum Patellarklonus ist es indessen nicht gekommen. Die Achillessehnenreflexe blieben erloschen.

Besonders bemerkenswert war das Verhalten der Fusssohlenreflexe. Bis zum Jahre 1901 waren sie nicht auszulösen. Zur Zeit, als Babinski auf die Wichtigkeit der reflektorisch auftretenden Dorsalflexion der Grosszehe bei taktilen Reizen der Fusssohle aufmerksam machte (1898), wurde auch Frau E. wie sämtliche Kranken der Anstalt wiederholt auf das eventuelle Vorhandensein dieses diagnostisch wertvollen Phänomens untersucht. Der Babinskische Reflex liess sich jedoch damals nicht feststellen. Erst später (12. XI. 1901) gelang sein Nachweis zum ersten Male und zwar nur am rechten Fusse, anfänglich auch nicht konstant, sondern nur, nachdem die Kranke längere Zeit ausser Bett und wiederholt im Zimmer auf und ab gegangen war. Nach mehrstündiger Bettruhe trat bei taktilen Reizen der Fusssohle die reflektorische Dorsalflexion der Grosszehe nicht auf. Später verschwand das Phänomen für mehrere Wochen wieder gänzlich, um schliesslich vom Frühjahr 1902 an zu einem dauernden, bis zu dem 'lode der Kranken nachweisbaren Symptome zu werden. Am linken Fuss trat das Babinskische Phänomen zum ersten Male im Juli 1902, anfangs auch nur 
als passagere Erscheinung, auf; und hier ist es im Laufe der Jahre zu einer habituellen Dorsalkontraktur der Grosszehe gekommen.

Die Druckschmerzhaftigkeit der Nervenstämme an den Extremitäten blieb, von Schwankungen in der Intensität abgesehen, unverändert. Im Sommer 1907 wurde vorübergehend auch eine Druckempfindlichkeit an der Austrittsstelle des N. infraorbitalis auf beiden Seiten beobachtet. Die Sensibilität war in der Hauptsache ungestört; vorübergehend liess sich eine Hypaesthesie am rechten Arm bei gleichzeitiger Thermohyperalgesie (1900) und eine Hyperalgesie auf der linken Körperhälfte nachweisen, während 1898 und 1899 der rechte Arm für Schmerzreize empfindlicher gewesen war und damals eine allgemeine leichte Hyperaesthesie auf der ganzen rechten Körperseite vorhanden zu sein schien.

Die Bauchreflexe blieben ständig erloschen. Ein Fehlen des Rachenreflexes liess sich erst 1903 nachweisen.

Schon im Jahre 1898 (vielleicht auch schon 1882 - passager (?): jedenfalls 1895) war ein Zittern beider Hände vorhanden, das bei intendierten Bewegungen nicht zunahm. Erst im Frühjahr 1903 fiel beim Essen, wozu die Kranke wegen der hochgradigen Lähmung des rechten Armes die linke Hand gebrauchte, ein stärkeres Zittern derselben auf, das um so stärker wurde, je melir die Kranke den Löffel dem Munde näherte. Auch bei dem Versuch, mit dem Zeigefinger der linken Hand die Nasenspitze oder einen vorgehaltenen Gegenstand $\mathrm{zu}$ berühren, liess sich jetzt ein unverkennbarer Intentionstremor wahrnehmen. Aber auch diese Erscheinung trug anfangs einen passageren Charakter und konnte erst im letzten Jahre vor dem Tode der Kranken zu den dauernd vorhandenen Symptomen gezählt werden.

Die Sprache der Kranken ist in dem Gutachten vom 27. VI. 1895 als ,verlangsamt" und ,schleppend" bezeichnet worden. Sie war zweifellos durch ein ausserordentlich defektes Gebiss erschwert; eine ausgesprochene Sprachstörung (Skandieren, Silbenstolpern oder dergl.) liess sich jedoch wälrrend der ganzen Dauer der Beobachtung der Kranken im Siechenhause nicht nachweisen.

Im Jahre 1898 bestand vorübergehend eine Andeutung von Nystagmus. Erst im November $1900 \mathrm{kam}$ dieses Symptom wieder zur Beobachtung und seit jener Zeit liess es sich gelegentlich immer wieder einmal in einwandfreier Weise feststellen. Zu anderen Zeiten aber fehlte der Nystagmus vollständig und ist auch bis zum Tode der Kiranken niemals längere Zeit hintereinander regelmässig beobachtet worden.

Augenhintergrund und Gesichtsfeld blieben jahrelang vollkommen normal. Erst im Dezember 1903 liess sich eine geringe konzentrische Einengung desGesichtsfeldes auf dem rechten Auge und eineetwasstärkere, unregelmässige Gesichtsfeldeinengung auf dem linken Auge nachweisen. Die Papille des rechten Auges zeigte erst im April 1907 eine beginnende Abblassung an ihrer temporalen Seite, die in der Folgezeit nur in geringem Masse zunahm, während der Augenhintergrund links keine Veränderung aufwies. Die Sehschärfe beider Augen blieb bis zuletzt gut.

Mitte November 1907 trat wiederum plötzlich eine Verschlimmerung in dem Zustand der Kranken ein. In der Nacht vom 17. zum 18. XI. brach sie bei dem Versuch, zum Nachtstuhl zu gehen, vor ihrem Bett zusammen, ohne das Bewusstsein zu verlieren, weil ihr die Beine plötzlich versagten. Am nächsten Morgen war sie unfähig, aufzustehen und zu gehen. Sie klagte über Kopfschmerz und stärkere Schmerzen im Rücken und in den Beinen. In den folgenden Tagen wurde wiederholt eine Inkontinenz der Blase beobachtet. Objektiv liess sich keine Aenderung in dem Zustand der Kranken nachweisen. Nach wenigen Tagen hatte sie sich wieder soweit erholt, dass sie am 21. November das Bett verlassen konnte.

Am 24. XI. erkrankte Frau E. unter mässigem Fieber an einer anscheinend leichten Influenza. Bald jedoch trat eine Bronchopneumonie im linken Unterlappen, eine doppelseitige purulente Otitis media und eine Diphtherie der Vaginal- und Urethralschleimhaut hinzu und unter den Erscheinungen einer allgemeinen Sepsis verstarb die Kranke am Vormittag des 9. XII. 1907. 
Der weitere Verlauf des Leidens und namentlich die Beobachtungen aus den Jahren 1901 bis 1903 liessen die anfänglich von mir gestellte Diagnose ,periphere Neuritis" unhaltbar erscheinen und haben mich schliesslich zu der Annahme geführt, dass dem proteusartigen Krankheitsbilde eine multiple Sklerose zugrunde liegen müsse. Unter dieser Diagnose habe ich die Kranke in dem ärztlichen Fortbildungskursus am 2. XII. 1903 vorgestellt. Die Diagnose stützte sich auf das Hinzutreten von spastischen Phänomenen in den gelähmten Extremitäten zu der bereits vorhandenen Muskelatrophie und auf den Nachweis der Babinskischen Dorsalflexion der Grosszehe, woraus geschlossen werden musste, dass sich der Krankheitsprozess gleichzeitig im peripheren und im zentralen Neuron der kortikomuskulären Bahn abspielte, während das Vorhandensein von Störungen der Sensibilität (Schmerzen, Paraesthesien etc.) erkennen liess, dass der Prozess nicht auf die motorische Bahn beschränkt war. Durch das Auftreten des Nystagmus und Intentionstremors und die Abblassung der temporalen Hälfte der Sehnervenpapille wurde schliesslich die Diagnose gesichert, mit der auch das psychische Verhalten der Kranken (Euphorie und leichte Demenz), sowie das anfänglich meist passagere Auftreten der einzelnen Symptome und der Verlauf des Leidens in Schüben in vollem Einklang stand.

Durch das Ergebnis der Obduktion und der histologischen Untersuchung des Zentralnervensystems ist die Diagnose bestätigt worden. Es fand sich neben der Bronchopneumonie im linken Unterlappen und einer frischen Lungenembolie im Hauptast der rechten A. pulmonalis eine typische multiple Sklerose des Gehirns und Rückenmarks. Schon mit dem blossen Auge waren auf zahlreichen Schnitten durch das Grosshirn, den Hirnstamm und das Rückenmark die derberen Herde $z u$ erkennen, die sich an der Luft bald graurot färbten. Doch liessen sich bei reicher Verbreitung in Pons, Oblongata und Rückenmark nur einzelne Herde im Gehirn auffinden, und auch histologisch waren keine Veränderungen in der Hirnrinde nachweisbar. Bei der mikroskopischen Bearbeitung zeigte es sich, dass die sklerotischen Herde in der ganzen Länge des Marks zerstreut und in der grauen wie weissen Substanz ganz unregelmässig lokalisiert waren. In einzelnen Höhen waren sie auf die Seitenstränge beschränkt, ohne indessen das Areal der Pyramidenseitenstränge scharf einzuhalten; vielmehr reichten sie auf verschiedenen Schnitten auch in die Vorder- und in die Kleinhirnseitenstränge hinein und vereinzelte Herde waren auch in den Hintersträngen vorhanden. In anderen Höhen, wie namentlich in der Hals- und Lendenanschwellung, griffen die sklerotischen Herde von dem Seitenstrang aus verschieden weit bald ein-, bald doppelseitig auf die grauen Vordersäulen und in besonderer Häufigkeit in das Gebiet der Wurzeleintrittszonen und Hinterhörner über.

Am unteren Ende des Duralsackes fanden sich zwischen den Lamellen der Dura liegend, bezw. deren Innenfläche durchsetzend, 
drei erbsengrosse, flache Tumoren, die sich bei der histologischen Untersuchung als Fibrome erwiesen.

An zahlreichen peripheren Nerven (Nn. ulnaris, radialis, medialis etc.) war eine Degeneration der Nervenfasern vorhanden.

Durch den anatomischen Befund ist die vier bis fünf Jahre vor dem Tode der Kranken gestellte Diagnose bestätigt und die frühere Annahme einer Polyneuritis als unrichtig erwiesen worden. Damit war zugleich auch die ursprüngliche Auffassung des Falles, die in der Einheitlichkeit der somatischen und psychischen Krankheitserscheinungen gipfelte und in der Diagnose der polyneuritischen Psychose zusammengefasst wurde, unhaltbar geworden. Es war deshalb von dem durch die gesicherte Diagnose gewonnenen neuen Standpunkte aus zu prüfen, ob sich an der Auffassung eines einheitlichen Krankheitsprozesses festhalten liesse, und die Frage zu erörtern: Ist es überhaupt ein und derselbe Krankheitsprozess gewesen, der im Jahre 1882 begonnen und bis $190 \%$ gedauert hat, oder sind nicht vielmehr verschiedene Krankheitsprozesse unabhängig von einander zur Entwicklung gekommen?

Zur Beantwortung dieser Frage sei daran erinnert,

dass die Schmerzen im rechten Arm, mit denen das Leiden begonnen hat, während dessen ganzer Dauer - nach den Angaben der Kranken - mit kurzen Unterbrechügen in wechselnder Stärke fortbestanden haben,

dass auch die ersten motorisehen Lähmungserscheinungen (Muskelatrophie und Parese), die nach dem anatomischen Befund (sklerotische Herde in den Vordersäulen des Halsmarks) mit Sicherheit auf die multiple Sklerose bezogen werden können, am rechten Arm zur Beobachtung gekommen sind,

und dass gleichzeitig mit der Ausbreitung der Schmerzen auf die anderen Extremitäten auch in diesen motorische Lähmungserscheinungen aufgetreten sind.

Diese Umstände und die besondere Häufigkeit der sklerotischen Plaques im Gebiet der Wurzeleintrittszonen und Hinterhörner des Hals- und Lendenmarks wiesen auf die Moglichkeit hin, dass die schon lange Jahre vor dem Auftreten der Muskelatrophie und der Paresen vorhandenen Schmerzen Initialsymptome der multiplen Sklerose (Wurzelschmerzen) gewesen seien.

Andererseits finden sich die Krankheitserscheinungen, die mit Sicherheit auf die multiple Sklerose zu beziehen sind, erst in den Gutachten aus dem Jahre 1895 verzeichnet, wozu freilich bemerkt werden muss, dass aus der Zeit zwischen 1891 und 1895 keine ärztlichen Untersuchungsbefunde vorliegen. So lässt sich also die Frage, ob die multiple Sklerose schon 1882 oder erst zwischen 1891 und 1895 begonnen hat, nicht mit Sicherheit und in einwandfreier Weise beantworten.

Hieraus ergibt sich auch die Unmöglichkeit eines sicheren Entscheides, ob die langjährige psychische Störung, die dem Auf- 
treten der motorischen Lähmungserscheinungen vorausgegangen ist, schon als Symptom der beginnenden multiplen Sklerose oder als selbständige Geisteskrankheit aufgefasst werden muss. Dass eine organische Erkrankung des Zentralnervensystems (multiple Sklerose) der psychischen Störung zugrunde gelegen hat, ist indessen zum mindesten unwahrscheinlich, weil gerade im Gehirn nur verhältnismässig wenige und kleine sklerotische Herde vorhanden und auch histologische Hirnrindenveränderungen nicht aufzufinden gewesen sind. Zudem pflegt die multiple Sklerose meines Wissens wohl regelmässig zu einer mit Euphorie verbundenen, fortschreitenden einfachen Demenz, nicht aber zu anderen psychischen Alterationen (Halluzinationen, Wahnideen, manische Erregungszustände, Stupor) zu führen, wie sie im Beginn der Psychose bei Frau E. beobachtet worden sind.

Wahrscheinlicher ist es deshalb, dass es sich um eine selbständige (funktionelle) Geisteskrankheit gehandelt hat. Wie aus den Akten erhellt, ist die psychische Störung in unmittelbarem Anschluss an eine Periode heftiger Schmerzen zum Ausbruch gekommen. Sie hat in ihrem akuten Stadium, dessen Dauer etwa ein Jahr betragen haben mag, unter lebhaften Sinnestäuschungen und Wahnideen schreckhaften Inhalts (Todesgedanken, Selbstmordversuche) zu einer depressiven, ängstlichen Stimmung und zu einem apathischen Verhalten der Kranken geführt, das nur vorübergehend durch expansive Erregungszustände unterbrochen wurde. Sie hat ferner frühzeitig eine hochgradige geistige Schuäche zur Folge gehabt. In ihrem späteren Verlanfe, der sich über mehrere Jahre ausgedehnt hat, hat die Psychose in unverkembarer Weise einen manisch-depressiven Typus gezeigt, und zwar noch zu einer Zeit, in der die sicheren somatischen Anzeichen der multiplen Sklerose manifest geworden sind.

Bei diesem Verlauf der geistigen Störung und angesichts des Umstandes, dass wir das Vorhandensein leichterer Krankheitserscheinungen manisch-depressiver Art vor dem Ausbruch der schweren Psychose nicht mit voller Sicherheit auszuschliessen vermögen, liesse sich vielleicht daran denken, dass es sich nm ein zirkulares Irresein gehandelt haben könnte, und dass die heftigen, monatelang anhaltenden Schmerzen bei der hochgradig anämischen Kranken einen besonders intensiven Anfall der primären Geisteskrankheit ausgelöst haben. Gegen diese Annahme scheint indessen der Umstand zu sprechen,

dass in dem von dem langjährigen Hausarzt der Kranken sorgfältig ausgefuilten Fragebogen eine erbliche Belastung negiert wird,

dass von dem Arzte vor dem Ausbruch der Psychose manischdepressive Krankheitserscheinungen nicht beobachtet worden sind,

dass auch von uns in den letzten Jahren keinerlei Schwankungen in der Stimmungslage der Kranken mehr wahrgenommen werden konnten, 
und schliesslich, dass im Verlauf der Psychose sehr frühzeitig eine geistige Schwäche in die Erscheinung getreten ist.

So bleibt zuletzt die Möglichkeit der Dementia praecox zu erwägen. Mit dem Verlauf dieser Geisteskrankheit ist die in den Akten enthaltene, kurze Schilderung des Beginns und des akuten Stadiums der Psychose schliesslich noch am besten in Einklang zu bringen, zumal die Dementia praecox in ihrem weiteren Verlauf auch einmal das Bild des manisch-depressiven Irreseins zeigen kann und regelmässig in einen Defektzustand übergeht, wie er bei Frau E. in späteren Jahren beobachtet worden ist.

Bei Abwägung dieser verschiedenen 'Möglichkeiten, deren keine freilich mit voller Sicherheit zu erweisen oder auszuschliessen sein wird, ist es am wahrscheinlichsten, dass in dem geschilderten Fall die psychische Störung (Dementia praecox) und die multiple Sklerose des Gehirns und Rückenmarks unabhängig von einander als selbständige Krankheitsprozesse verlaufen sind. Für diese Annahme spricht,

dass in den ersten 9 (bis 13) Jahren nach Ausbruch der Psychose irgendwelche Erscheinungen, die mit Sicherheit auf die multiple Sklerose $\mathrm{zu}$ beziehen gewesen wären, gefehlt haben,

dass leichte Krankheitserscheinungen manisch-depressiver Art, wie sie der multiplen Sklerose nicht eigen zu sein pflegen, noch zu einer Zeit vorhanden gewesen sind, in der die ersten sicheren Anzeichen einer organischen Erkrankung des Zentralnervensystems manifest wurden,

und dass die Demenz, die zwischen 1895 und 1897 stationär geblieben war (Defektzustand nach Ablauf der Dementia praecox), in den letzten neun Jahren wieder aufs neue progressiv gewesen ist (im Verlauf der multiplen Sklerose).

Die beiden letzten Umstände sprechen zugleich dafür, dass die funktionelle Psychose und die organische Erkrankung des Gehirns und Rückenmarks auch zeitlich noch nebeneinander verlaufen sind. Und gerade dieses zeitliche Zusammenfallen psychischer Anomalien, die durch die Dementia praecox bedingt gewesen sein mögen (Demenz und leichte manische Erregung), mit den somatischen Erscheinungen der organischen Erkrankung des Zentralnervensystems (Schmerzen, Muskelatrophie und Parese) haben die Erkenntnis der wahren Natur des Leidens lange Zeit hindurch ausserordentlich erschwert, so dass irriger Weise 1895 eine progressive Paralyse, 1899 eine polyneuritische Psychose angenommen worden ist, bis schliesslich das Hinzutreten eindeutiger Symptome in den Jahren 1901 bis 1903 die durch den Obduktionsbefund bestätigte Diagnose ermöglicht hat. 